\title{
A Life Prediction Model for Structure under HCF-LCF Interaction Loading
}

\author{
LIN Wenjuan ${ }^{1, a^{*}}$ and HONG Haiming ${ }^{2, b}$ \\ ${ }^{1}$ Institute of Solid Mechanics, Beihang University, Beijing 100191, PR China \\ ${ }^{2}$ Shenyang aircraft design and research institute, Shenyang 110035, PR China \\ a327216844@qq.com, bhhmzhs@hotmail.com
}

Keywords: HCF-LCF, fatigue life, life prediction model.

Abstract. A life prediction model for HCF-LCF interaction loading which comprehensively considers the ratio of the HCF load to the LCF load and the ratio of stress amplitude in this work. The prediction model based on the Miner model, but correct its error by considers that the loads lower than the fatigue limit stress which contribute to fatigue damage. The prediction model has the advantage of uncomplicated calculation and simple form. Moreover it can get a better result in prediction effect for HCF-LCF interaction loading problem.

\section{Introduction}

Fatigue failure is a typical failure mode which widely exists in the aircraft, engine and other metal structure $^{[1-3]}$. According to ASTM E1823-13 ${ }^{[4]}$, fatigue refers to a development process which in a point or some points to bear stress perturbations and after a sufficient number of cyclic disturbance is formed crack or disturbance stress, high localized stress crack. Fatigue problem can be classified into HCF (high cycle fatigue) problem and LCF (low cycle fatigue) problem. HCF means the fatigue phenomenon that the material life is in the range of $10^{4}-10^{5}$ cycles under low stress amplitudes. It does not generate significant plastic deformation and is usually studied based on stress analysis method. Differently, LCF means the fatigue phenomenon that material life is lower than the range of $10^{4}-10^{5}$ cycles under high amplitudes. It usually generates obvious plastic deformation and is usually studied through strain analysis method.

The fatigue load, caused by airflow during a fight, is not invariable. Hence, the fatigue problem does not belong to pure LCF or pure HCF, but the combination of LCF and HCF, i.e., the high-cycle and low-cycle interaction fatigue. The reason is that the plane structure not only suffers the LCF with high load and low frequency caused by centrifugal force from the engine, but also the HCF with low load and high frequency caused by the air resistance. The existing researches ${ }^{[5-7]}$ show that fatigue life of plane structure is greatly reduced under the effect of the compound load of LCF and HCF. Usually, researches employ the linear cumulative damage models to analyze the experiment of the HC-LC interaction loading. Among these models, the Miner model based on the assumption of symmetrical cycle and equal damage is most commonly used. Its errors can be neglected when stress amplitude is low and material toughness is enough. Moreover, due to the simple form and convenient usage, the Miner model has been wide used in analysis of fatigue problem. Nevertheless, Miner model considers that the loads lower than the fatigue limit stress rarely contribute to fatigue damage, which consequently leads to a great flaw in analyzing the fatigue problem under the HC-LC interaction load of which the HCF load of is lower than the fatigue limit stress.

In this paper, we modify the Miner ${ }^{[8]}$ model through HCF-LCF interaction loading based experiment study. We propose a new model to predict fatigue life, which comprehensively considers the ratio of the HCF load to the LCF load and the ratio of stress amplitude under HCF-LCF interaction loading. Additionally, our model provides valuable insight into the analysis of fatigue life especially under HCF-LCF interaction loading. 


\section{Model modification under HCF-LCF interaction loading}

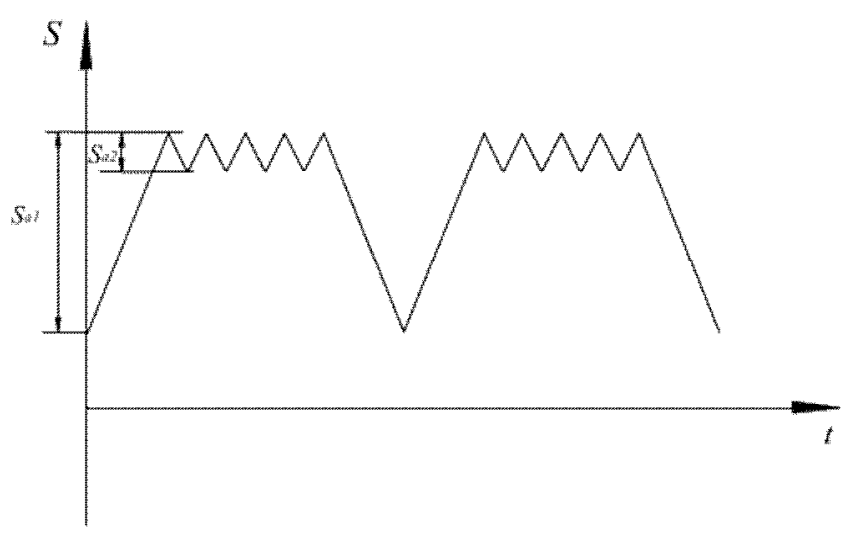

Fig. 1 The sketch map of load spectrum

According to the Miner model for a given specimen, the fatigue life under constant stress amplitude as $S_{a 1}$ is $N_{1}$. Therefore $n_{1}$ loading cycles are applied, the fatigue life of the specimen is reduced by $n_{1} / N_{1}$. It is obvious that when $n_{1} / N_{1}=1$, the specimen is fractured proportion of the fatigue resistance. Here, $N_{1}$ corresponds to the fatigue life of a specimen stressed with amplification as $S_{a 1}$. The equation of Miner model can be extended to $\sum \frac{n_{i}}{N_{i}}=1$ if load spectrum includes two and more kinds of stress amplitudes. Focus on the case that the load spectrum has only two kinds of stress amplitude as $S_{a 1}$ and $S_{a 2}$ ( as Fig. 1 ), $S_{a 2}$ is lower than the fatigue limit stress. We then considered the fatigue life $N_{2}$ under constant stress amplitude as $S_{a 2}$ is infinite, i.e., $n_{2} / N_{2}=0$, which means $\sum_{i=1}^{2} \frac{n_{i}}{N_{i}}=1$ cannot be satisfied. Therefore, the fracture of specimen would never occur according to the Miner model ${ }^{[9]}$. However, present experiments found that, while different stress amplitudes which can either are higher or lower than the fatigue limit stress simultaneously exit in the same load spectrum, the fatigue life of the specimen will be significantly reduced.

In order to propose a new model to predict fatigue life, which comprehensively considers the ratio of the HCF load to the LCF load and the ratio of stress amplitude under HCF-LCF interaction loading. First let us begin from the principle of the Miner model. The damage caused by a cycle of load spectrum can be expressed as

$$
D=\frac{1}{N}
$$

A cycle block of load spectrum causes the amount of damage as follows

$$
D_{1}=\frac{n}{N_{H}}+\frac{1}{N_{L}}
$$

Therefore, the damage caused by $N$ cycle blocks of load spectrum holds as

$$
D_{N}=N D_{1}=N\left(\frac{n}{N_{H}}+\frac{1}{N_{L}}\right)
$$


When the total damage reaches to 1 , the specimen is broken. Denote this broken life as $N_{c}$, i.e.,

$$
D_{c r}=N_{c}\left(\frac{n}{N_{H}}+\frac{1}{N_{L}}\right)=1
$$

However, while observing from the experiments, the HCF-LCF interaction loading damage is not the simple superimposition of HCF damage and LCF damage. The HCF damage is obviously higher than $\frac{n}{N_{H}}$, and is related to the ratio of stress amplification $\alpha$ and the ratio of the number of cycles $n$ under HC-LC interaction loading. Further, a correction model should satisfy the following bounding conditions.

a) If the ratio of the number of load spectrum cycles $n=0$, the analytical life should be equal to the life of pure LCF $N_{L}$.

b) If the ratio of load spectrum stress amplification $\alpha=0$, the analytical life should also be equal to $N_{L}$.

c) If the ratio of load spectrum stress amplification $\alpha=1$, the analytical life should also be equal to $(n+1) N_{L}$.

In order to correctly reflect the damage caused by $\mathrm{HCF}$, we let $D_{H}=\frac{\lambda}{N_{H}}$, where $\lambda$ is a parameter related to the ratio of HCF-LCF stress amplification $\alpha$ and the ratio of the number of HCF-LCF load spectrum cycles $n$. Considering the bounding conditions mention above, we can let $\lambda=\alpha^{m} n$. Hence, the prediction model is obtained as follows

$$
D_{N}=N\left(\frac{\alpha^{m} n}{N_{H}}+\frac{1}{N_{L}}\right)
$$

where $m$ can be determined through fitting the experiment data.

The rationality of our prediction model is validated by the bounding conditions as follows.

a) If the ratio of the number of load spectrum cycles $n=0$, the analytical life $N_{c}$ satisfies $D_{c r}=N_{c}\left(0+\frac{1}{N_{L}}\right)=1$. Thus $N_{c}=N_{L}$.

b) If the ratio of load spectrum stress amplification $\alpha=0$, the analytical life $N_{c}$ satisfies $D_{c r}=N_{c}\left(0+\frac{1}{N_{L}}\right)=1$. Therefore, $N_{c}=N_{L}$.

c) If the ratio of load spectrum stress amplification $\alpha=1$, the analytical life $N_{c}$ satisfies $D_{c r}=N_{c}\left(\frac{n}{N_{H}}+\frac{1}{N_{L}}\right)=1$. Since $\alpha=1$, the HCF life equals to the LCF life, i.e., $N_{H}=N_{L}$. We consequently have $N_{c}=(1+n) N_{L}$.

In summary, our prediction model is proved to satisfy the three bounding conditions. 


\section{Verification of the model}

In the previous subsection, we have proposed our prediction model and verified the rationality of it through the bounding conditions. In this subsection, we will apply the experiment data from Li Rui' research ${ }^{[10]}$ to shown that the model is accurate. Experiment life result of pure LCF with stress level $190 \mathrm{Mpa}$ and stress ratio 0.1 is 38163 , namely $N_{L}=38163$. The other experiment data is depicted as Table 1, Table 2.

Table 1 Result of pure HCF experiment

\begin{tabular}{cccccc}
\hline$\alpha$ & 0 & 0.1 & 0.2 & 0.3 & 0.5 \\
\hline$N_{H}$ & $\infty$ & $\infty$ & $\infty$ & $1,057,528$ & 173,833 \\
\hline
\end{tabular}

Table 2 Result of HCF-LCF interaction experiment under different cycle number ratio and different stress amplitude ratio

\begin{tabular}{ccccc}
\hline$n$ & $\alpha$ & Mean life & Logarithmic life & C.V. \\
\hline 0 & 0.2 & 38,163 & 4.582 & 0.016 \\
\hline 5 & 0.2 & 30,472 & 4.484 & 0.014 \\
\hline 10 & 0.2 & 27,292 & 4.436 & 0.004 \\
\hline 30 & 0.2 & 17,842 & 4.251 & 0.012 \\
\hline 60 & 0.2 & 15,854 & 4.200 & 0.011 \\
\hline 10 & 0 & 38,163 & 4.582 & 0.016 \\
\hline 10 & 0.1 & 29,027 & 4.463 & 0.005 \\
\hline 10 & 0.3 & 15,038 & 4.177 & 0.008 \\
\hline 10 & 0.5 & 7,027 & 3.847 & 0.021 \\
\hline
\end{tabular}

C.V.: coefficient of variation

We use the least square method to determine the optimal value of the fitting parameter $m=-0.6902$, i.e., the closed form of the prediction model holds as $D_{N}=N\left(\frac{\alpha^{-0.6902} n}{N_{H}}+\frac{1}{N_{L}}\right)$. Putting the experiment data into the prediction model, the analytical life are obtained and shown in Table 3.

Table 3 Analytical result of prediction model

\begin{tabular}{ccccc}
\hline$n$ & $\alpha$ & $\begin{array}{c}\text { Experimental } \\
\text { logarithmic life }\end{array}$ & $\begin{array}{c}\text { Prediction } \\
\text { logarithmic life }\end{array}$ & Deviation \\
\hline 0 & 0.2 & 4.582 & 4.582 & $0.00 \%$ \\
\hline 5 & 0.2 & 4.484 & 4.499 & $-0.33 \%$ \\
\hline 10 & 0.2 & 4.436 & 4.429 & $0.16 \%$ \\
\hline 30 & 0.2 & 4.251 & 4.226 & $0.59 \%$ \\
\hline 60 & 0.2 & 4.200 & 4.034 & $3.96 \%$ \\
\hline 10 & 0 & 4.582 & 4.582 & $0.00 \%$ \\
\hline 10 & 0.1 & 4.463 & 4.577 & $-2.56 \%$ \\
\hline 10 & 0.3 & 4.177 & 4.320 & $-3.41 \%$ \\
\hline 10 & 0.5 & 3.847 & 3.924 & $-2.02 \%$ \\
\hline
\end{tabular}




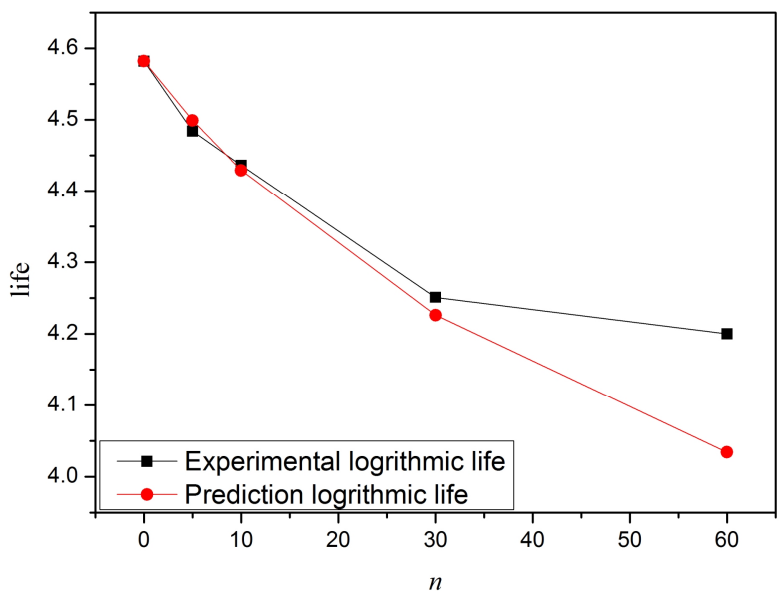

(a) Life vs $n$

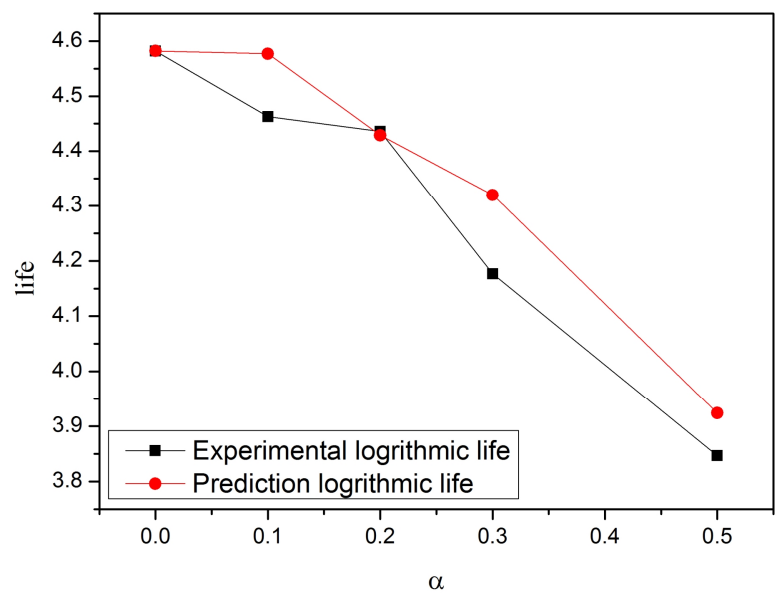

(b) Life vs $\alpha$

Fig. 2 Analytical result of prediction model

Table 3 and Fig. 2 indicates that the variation trend of the analytical life conforms to the one of experiment life. Also, the gaps between the analytical life and experiment life are all within one times of life.

\section{Conclusions}

This paper has proposed a prediction model to well improve the Miner model. The advantages of the prediction model address not only its simple form and ease of use, but also comprehensively taking the ratio of the number of load spectrum cycles and the ratio of load spectrum stress amplification in to account while studying the impact of fatigue life under HCF-LCF interaction loading. The model extends the applicable range of the cumulative damage theory and provides valuable insight into life prediction of material.

\section{References}

[1] J. Zhang, X. Shi, R. Bao, B. Fei. Int. J. Fatigue, 33 (2011), 1066-1074.

[2] J. Zhang, X. Shi, B. Fei. Int. J. Fatigue, 38 (2012), 144-154.

[3] J. Zhang, Q. Xiao, X. Shi, B. Fei. Int. J. Fatigue, 67 (2014), 173-182.

[4] ASTM E1823-13. American Society for Testing of Materials, West Conshohocken, PA, USA; 2013.

[5] D. Gelmedin, K.H. Lang. Procedia Engineering, 2 (2010) 1343-1352.

[6] B.E. Powell, T.V. Duggan. Int. J. Fatigue, 9 (1987) 195-202.

[7] J. Byrne, R.F. Hall, B.E. Powell. Int. J. Fatigue, 25 (2003) 827-834.

[8] M.A. Miner. J. Appl. Mech., 67 (1945) 159-164.

[9] J. Schijve. Springer Netherlands, 2001, 238-239.

[10] R. Li. Aircraft Design, 30 (2010) 18-22. 